\title{
Development of an Integrated Control of Front Steering and Torque Vectoring Differential Gear System Using Modelica
}

\author{
Yutaka Hirano $^{1}$ \\ ${ }^{1}$ Toyota Motor Corporation, Japan, yutaka_hirano@mail.toyota.co.jp
}

\begin{abstract}
To achieve future low carbon mobility society, many new-type electric vehicles (EVs) are developed actively in recent period. Those EVs have integrated power unit which take place of conventional engine, transmission and differential gear components. Additionally it is rather easy to integrate torque vectoring function to those power units using gear sets to control torque distribution between left wheel and right wheel. In this paper, model-based development of an integrated control of the front steering angle and torque vectoring differential (TVD) gear system is described. New integrated control logic was developed using model matching control to let the vehicle yaw rate and vehicle slip angle follow the desired dynamics. Simulation results using an extended single track model of vehicle dynamics are shown to prove the efficacy of the proposed control. Though, full vehicle model considering all of vehicle dynamics and drive train motion using Modelica clarified the problem of this method in actual cases. Difference between the extended single track model and full vehicle model was compared to estimate the reason of the problem.
\end{abstract}

Keywords: $\quad$ Model Based Development, Vehicle Dynamics, Torque Vectoring, Model Matching Control

\section{Introduction}

To satisfy needs for future low-carbon mobility society, development of many new EVs is increasingly active in recent years. Additionally many new proposals about integrated electric power train which also has torque vectoring capability are presented (Höhn et al., 2013). (Burgess, 2009) showed a model-based control design of TVD using an inverse model for feedforward control. (Efstathios et al. 2015) introduced a model predictive control of TVD considering nonlinear tire characteristics. On the other hand, authors have researched a new control of TVD by using traditional PI feedback control (Hirano et al., 2013)(Hirano et al., 2014). The author also utilized a model matching control theory to develop a new control of TVD (Hirano, 2016a). Additionally the author expand the control to the integrated control of TVD and active front steering (AFS) by model matching control (Hirano, 2016b). The purpose of using both TVD and AFS is to control both vehicle yaw rate and slip angle independently. In the last paper, the derived control was based on simple LQR (Linear Quadratic Regulator) and there was no measure to cope with steady state deviation. In this paper, the LQR design was modified by augmenting the plant model to include integral of the state variables. As same as the last research, an extended single track model of vehicle dynamics was used to derive and verify the new control. Finally the developed control was verified by using the full vehicle model using Modelica. Some measures about solving problems when applying Modelica to this kind of problem are also mentioned.

\section{Experimental EV}

Table 1 Specifications of new experimental EV

\begin{tabular}{|l|l|l|}
\hline & New EV & $\begin{array}{l}\text { Conventional } \\
\text { car }\end{array}$ \\
\hline Vehicle Mass & $750 \mathrm{~kg}$ & $1240 \mathrm{~kg}$ \\
\hline $\begin{array}{l}\text { Yaw Moment } \\
\text { Inertia }\end{array}$ & $869 \mathrm{kgm}^{2}$ & $2104 \mathrm{kgm}^{2}$ \\
\hline Wheelbase & $2.6 \mathrm{~m}$ & $2.6 \mathrm{~m}$ \\
\hline $\begin{array}{l}\text { Front : Rear Weight } \\
\text { Distribution }\end{array}$ & $0.48: 0.52$ & $0.62: 0.38$ \\
\hline Height of CG & $0.38 \mathrm{~m}$ & $0.55 \mathrm{~m}$ \\
\hline Tire RRC & $5 \times 10^{-3}$ & $8.8 \times 10^{-3}$ \\
\hline Tire Normalized CP & 16.1 & 20.4 \\
\hline
\end{tabular}

The proposed experimental EV has specifications as shown in Table 1. Compared with a conventional small-class passenger car, the new EV has better characteristics of lighter vehicle weight, smaller yaw moment of inertia, lower height of the center of gravity (CG) and lower rolling resistance coefficients (RRC) value of tires. Because of these characteristics, this new EV is expected to have better handling and lower energy consumption than conventional vehicles. On the other hand, because of lighter weight and lower value of tire normalized CP (Cornering Power), this new EV seems more sensitive against external disturbances such as crosswind and road irregularity than the conventional cars. To cope with this problem, direct yaw moment control (DYC) was applied by using a 
new integrated transaxle unit for rear axle which has a main electric motor and also TVD gear unit with a control motor. Additionally, to control both yaw rate and slip angle of the vehicle independently, another control input of active front steering (AFS) was introduced.

\section{Vehicle Model}

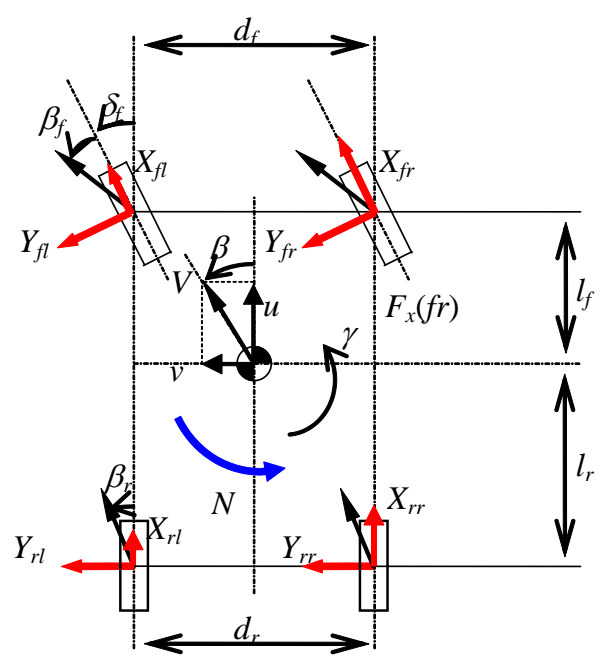

Figure 1. Extended single track vehicle model

Figure 1 shows an extended single track vehicle model to derive the control logic. Usually the single track model calculates front and rear tire side forces by adding both tire forces of right tire and left tire respectively. But in this paper, the model was extended to separate the tire longitudinal forces of right tire and left tire to consider direct yaw moment generated by the difference of the longitudinal forces of right tire and left tire. The coordinate system of this model follows FLU (x: forward, y: leftward, z; upward) convention. The simplified equations of motion by this extended single track model become as follows.

$$
\begin{gathered}
M \frac{d V}{d t}=F=\left(X_{r r}+X_{r l}\right) \\
M V\left(\frac{d \beta}{d t}+\gamma\right)=2 Y_{f}+2 Y_{r} \\
I_{z} \frac{d \gamma}{d t}=2 l_{f} Y_{f}-2 l_{r} Y_{r}+N
\end{gathered}
$$

where

$$
\begin{gathered}
Y_{f}=-K_{f} \beta_{f}=-K_{f}\left(\beta+\frac{l_{f}}{V} \gamma-\delta_{f}\right) \\
Y_{r}=-K_{r} \beta_{r}=-K_{r}\left(\beta-\frac{l_{r}}{V} \gamma\right) \\
N=d_{r}\left(X_{r r}-X_{r l}\right)
\end{gathered}
$$

$\beta \quad$ : Vehicle slip angle,

$\gamma \quad$ : Vehicle yaw rate,

$M \quad$ : Vehicle mass,

$V \quad$ : Vehicle velocity,

$I_{z} \quad$ : Vehicle yaw moment of inertia,

$l_{f}\left(l_{r}\right)$ : Distance from the CG to front (rear) axle, (CG: Center of Gravity)

$d_{f}\left(d_{r}\right)$ : Tread of front (rear) axle,

$X_{* *} \quad$ : Longitudinal force of each tire,

$Y_{f}\left(Y_{r}\right) \quad$ : Lateral force of front (rear) tires,

$\delta_{f} \quad$ : Steering angle of front tire,

$F \quad$ : Vehicle driving force,

$N \quad$ : Direct yaw control moment by TVD.

$K_{f}$ and $K_{r}$ are the equivalent cornering power of front and rear tire respectively.

If driving force $F$ and DYC moment $N$ can be calculated by some control logic, then the target longitudinal forces of left and right rear wheels to be realized by TVD power unit become as follows from Equation (1) and (6).

$$
\begin{aligned}
& X_{r r}=\frac{1}{2}\left(F+\frac{N}{d_{r}}\right) \\
& X_{r l}=\frac{1}{2}\left(F-\frac{N}{d_{r}}\right)
\end{aligned}
$$

\section{Control Design}

\subsection{Longitudinal Driving Force Control}

Let us suppose that the desired value of vehicle speed, vehicle yaw rate and vehicle slip angle are defined as $V_{\text {ref }}, \gamma_{\text {ref }}$ and $\beta_{\text {ref }}$ respectively.

The desired vehicle driving force $F$ can be calculated as below by PI feedback control and Equation (1)..

$$
F=M \frac{d V_{r e f}}{d t}+K_{P F}\left(V_{r e f}-V\right)+K_{I F} \int\left(V_{r e f}-V\right) d t
$$

Here $K_{P F}$ is a proportional feedback gain and $K_{I F}$ is an integral feedback gain.

\subsection{Model Matching Control of Lateral Dynamics}

For the lateral dynamics, the state space form of the vehicle dynamics with TVD and AFS control becomes as follow from Equations (2) and (3).

$$
\dot{x}=A x+B u
$$

Here,

$$
\begin{gathered}
x=\left[\begin{array}{l}
\beta \\
\gamma
\end{array}\right]: \text { State variables } \\
u=\left[\begin{array}{l}
\delta_{f} \\
N
\end{array}\right]: \text { Control inputs }
\end{gathered}
$$

Here, 


$$
\begin{gathered}
A=\left[\begin{array}{rr}
-\frac{2\left(K_{f}+K_{r}\right)}{M V} & -1-\frac{2\left(l_{f} K_{f}-l_{r} K_{r}\right)}{M V^{2}} \\
-\frac{2\left(l_{f} K_{f}-l_{r} K_{r}\right)}{I_{z}} & -\frac{2\left(l_{f}^{2} K_{f}+l_{r}^{2} K_{r}\right)}{I_{z} V}
\end{array}\right] \\
=\left[\begin{array}{ll}
a_{11} & a_{12} \\
a_{21} & a_{22}
\end{array}\right] \\
B=\left[\begin{array}{ll}
\frac{2 K_{f}}{M V} & 0 \\
\frac{2 l_{f} K_{f}}{I_{z}} & \frac{1}{I_{z}}
\end{array}\right]=\left[\begin{array}{ll}
b_{11} & b_{12} \\
b_{21} & b_{22}
\end{array}\right]
\end{gathered}
$$

Please note that the elements of the matrix A of the Equation (10) are dependent on the vehicle velocity $V$ as shown in the Equation (13). So the vehicle dynamics system described by the Equation (10) is a time-variant system.

It is well known that the response of both yaw rate and slip angle become to the second order lag function of the steering input when no control is applied. This fact results in that the ordinary drivers tend to respond to steer with time lag against the vehicle motion and tend to result in vehicle spin when the vehicle motion becomes unstable such as on the slippery road. On the other hand, it becomes easier for drivers to stabilize the vehicle if the response of the vehicle motion will become to the first order lag function, i.e. there is no resonance characteristics about the vehicle dynamics.

Thus, the desired dynamics of vehicle yaw rate and vehicle slip angle are assumed as the first order lag function of the driver's steering wheel input, as shown by the Equation (15).

$$
x_{d}=\left[\begin{array}{l}
\beta_{r e f} \\
\gamma_{r e f}
\end{array}\right]=\left[\begin{array}{l}
\frac{k_{\beta}}{1+s \tau_{\beta}} G_{\beta 0} \\
\frac{k_{\gamma}}{1+s \tau_{\gamma}} G_{\gamma 0}
\end{array}\right] \delta_{s}
$$

Here, $s$ is Laplace operator. $k_{\beta}$ and $k_{\gamma}$ are gain of desired slip angle and desired yaw rate from the steady state gain of each state variables, while $G_{\beta 0}$ and $G_{\gamma 0}$ are steady state gain of the slip angle and the yaw rate respectively from the steering wheel input angle $\delta_{s}$. Also $\tau_{\beta}$ and $\tau_{\gamma}$ are time constant of the desired slip angle and the desired yaw rate as the first order lag function.

$G_{\beta 0}$ and $G_{\gamma 0}$ are calculated as follows. Considering the case of steady state as $x=x_{0}$ and without any active control, the Equation (10) becomes as bellow.

$$
\dot{x}_{0}=0=A x_{0}+E \delta_{s}
$$

where

$$
E=\left[\begin{array}{c}
\frac{2 K_{f}}{G_{s} M V} \\
\frac{2 l_{f} K_{f}}{G_{s} I_{z}}
\end{array}\right]
$$

Here, $G_{s}$ : steering gear ratio. From the Equation (16), $x_{0}$ is obtained as follow.

$$
\begin{aligned}
x_{0}= & -A^{-1} E \delta_{s} \\
=- & \frac{M I_{z} V^{2}}{4 K_{f} K_{r}\left(l_{f}+l_{r}\right)^{2}-2 M V^{2}\left(l_{f} K_{f}-l_{r} K_{r}\right)} \\
& \times\left[\begin{array}{c}
-\frac{4 K_{f} K_{r} l_{r}\left(l_{f}+l_{r}\right)}{M I_{z} V^{2}}+\frac{2 l_{f} K_{f}}{I_{z}} \\
\frac{-4 K_{f} K_{r}\left(l_{f}+l_{r}\right)}{M I_{z} V}
\end{array}\right] \frac{1}{G_{s}} \delta_{s}
\end{aligned}
$$

Thus, $G_{\beta 0}$ and $G_{\gamma 0}$ can be calculated as the following equation.

$$
\begin{aligned}
{\left[\begin{array}{c}
G_{\beta 0} \\
G_{\gamma 0}
\end{array}\right] } & =-\frac{M I_{z} V^{2}}{4 K_{f} K_{r}\left(l_{f}+l_{r}\right)^{2}-2 M V^{2}\left(l_{f} K_{f}-l_{r} K_{r}\right)} \\
& \times\left[\begin{array}{c}
-\frac{4 K_{f} K_{r} l_{r}\left(l_{f}+l_{r}\right)}{M I_{z} V^{2}}+\frac{2 l_{f} K_{f}}{I_{z}} \\
\frac{-4 K_{f} K_{r}\left(l_{f}+l_{r}\right)}{M I_{z} V}
\end{array}\right] \frac{1}{G_{s}}
\end{aligned}
$$

The model matching control can be derived as below. The state space form of the desired dynamics can be written as below from the Equation (15).

$$
\dot{x}_{d}=A_{d} x_{d}+E_{d} \delta_{s}
$$

Here,

$$
\begin{aligned}
A_{d} & =\left[\begin{array}{cc}
-\frac{1}{\tau_{\beta}} & 0 \\
0 & -\frac{1}{\tau_{\gamma}}
\end{array}\right] \text { and } \\
E_{d} & =\left[\begin{array}{l}
\frac{k_{\beta}}{\tau_{\beta}} G_{\beta 0} \\
\frac{k_{\gamma}}{\tau_{\gamma}} G_{\gamma 0}
\end{array}\right] .
\end{aligned}
$$

Assume the error between actual state variables and desired state variables as $e=x-x_{d}$. A dynamic state equation of this error variable can be obtained as below by subtracting Equation (20) from Equation (10).

$$
\dot{e}=A e+B u+\left(A-A_{d}\right) x_{d}-E_{d} \delta_{s}
$$

In the previous research (Hirano, 2016b), the simulation results of full vehicle model showed that there were some steady state deviation remained after stabilizing the vehicle motion. Thus, it is suggested that augmenting the state space equation of the Equation (21) to include the integral of the state variables is necessary. By assuming a new state vector of the error vector as 


$$
\hat{e}=\left[\begin{array}{c}
\beta-\beta_{\text {ref }} \\
\gamma-\gamma_{\text {ref }} \\
\int\left(\beta-\beta_{\text {ref }}\right) d t \\
\int\left(\gamma-\gamma_{\text {ref }}\right) d t
\end{array}\right]
$$

the Equation (21) is augmented as below.

$$
\begin{aligned}
& \dot{\hat{e}}= {\left[\begin{array}{cccc}
A & 0 & 0 \\
1 & 0 & 0 & 0 \\
0 & 1 & 0 & 0
\end{array}\right] \hat{e}+\left[\begin{array}{cc}
B & 0 \\
0 & 0
\end{array}\right] u } \\
&+\left[\begin{array}{cc}
\left(A-A_{d}\right) \\
0 & 0 \\
0 & 0
\end{array}\right] x_{d}-\left[\begin{array}{cc}
E_{d} \\
0 & 0 \\
0 & 0
\end{array}\right] \delta_{s} \\
& \stackrel{\text { def }}{=} \hat{A} \hat{e}+\hat{B} u+\widehat{A_{d e}} x_{d}-\widehat{E_{d e}} \delta_{s}
\end{aligned}
$$

Here,

$$
\begin{gathered}
\hat{A} \stackrel{\text { def }}{=}\left[\begin{array}{cccc}
A & 0 & 0 \\
1 & 0 & 0 & 0 \\
0 & 1 & 0 & 0
\end{array}\right]=\left[\begin{array}{ll}
A & O_{2} \\
I_{2} & O_{2}
\end{array}\right] \\
\widehat{B} \stackrel{\text { def }}{=}\left[\begin{array}{ll}
0 & 0 \\
0 & 0
\end{array}\right]=\left[\begin{array}{c}
B \\
O_{2}
\end{array}\right] \\
\widehat{A_{d e}} \stackrel{\text { def }}{=}\left[\begin{array}{cc}
\left(A-A_{d}\right) \\
0 & 0 \\
0 & 0
\end{array}\right]=\left[\begin{array}{c}
\left(A-A_{d}\right) \\
O_{2}
\end{array}\right] \\
\widehat{E_{d e}} \stackrel{\text { def }}{=}\left[\begin{array}{cc}
E_{d} \\
0 & 0 \\
0 & 0
\end{array}\right]=\left[\begin{array}{c}
E_{d} \\
O_{2}
\end{array}\right]
\end{gathered}
$$

where $O_{2}$ is the zero matrix of order 2 and $I_{2}$ is the unit matrix of order 2.

Let's assume a virtual control input of the augmented state space equation of the error vector as follow.

$$
\widehat{U} \stackrel{\text { def }}{=} \hat{B} u+\widehat{A_{d e}} x_{d}-\widehat{E_{d e}} \delta_{s}
$$

Then the Equation (23) becomes as follow.

$$
\dot{\hat{e}}=\widehat{A} \hat{e}+I_{4} \widehat{U}
$$

Here $I_{4}$ is the unit matrix of order 4 . For the linear system of the Equation (25), we can design a feedback control

$$
\widehat{U}=\widehat{K} \hat{e}
$$

by linear control theory. In this paper, the feedback gain $\hat{K}$ is calculated by using LQR (Linear Quadratic Regulator) so that the following criteria function is minimized.

$$
J=\int_{0}^{\infty}\left(\hat{e}^{T} Q \hat{e}+\hat{U}^{T} R \hat{U}\right) d t
$$

Here, $Q$ and $R$ are weight matrixes of order 4. Please note that $\hat{K}$ is dependent on vehicle velocity because the matrix $A$ included in $\hat{A}$ is velocity dependent as shown in Equation (13). Figure 2 shows some elements of feedback gain $\hat{K}$ as a function of the vehicle velocity $V$.

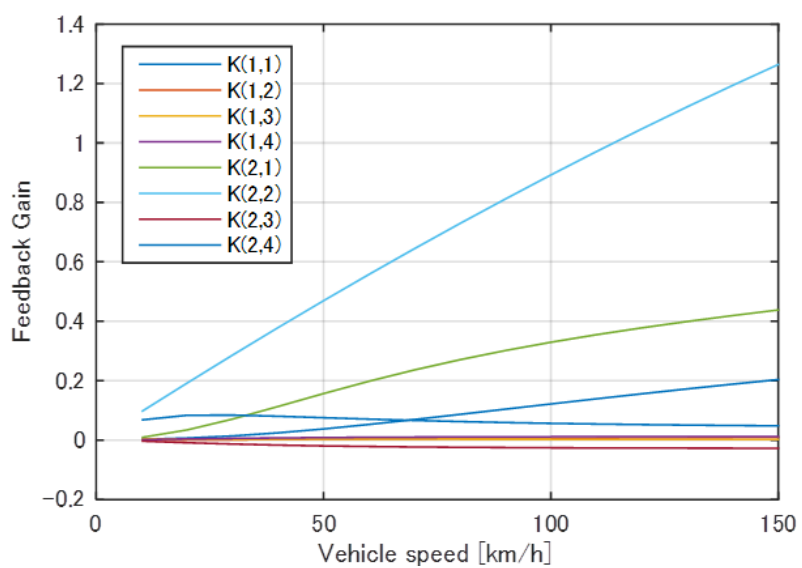

Figure 2. Plot of LQR gain according to vehicle speed

Finally the actual control input $u$ is calculated by the Equations (24) and (26) as below. At first the Equation (24) is rewritten as below.

$$
\left[\begin{array}{l}
\widehat{K_{1}} \\
\widehat{K_{2}}
\end{array}\right] \hat{e}=\left[\begin{array}{c}
B u+\left(A-A_{d}\right) x_{d}-E_{d} \delta \\
S
\end{array}\right]
$$

Here, $\widehat{K_{1}}$ is the upper part of size $(2 \times 4)$ of the gain matrix $\widehat{K}$ and $\widehat{K_{2}}$ is the lower part of size $(2 \times 4)$ of the gain matrix $\widehat{K} . S$ is an unknown variable.

From the upper part of the Equation (27) the actual control input $u$ can be calculated as below.

$$
u=B^{-1}\left\{-\hat{K}_{1} \hat{e}-\left(A-A_{d}\right) x_{d}+E_{d} \delta_{s}\right\}
$$

Here, $B^{-1}$ is the inverse matrix of $B .\left(B^{-1} B=I_{2}\right.$.) From the Equation (28) it is understood that the control input consists of a feedback term of the augmented state error and two feedforward terms of desired state variables and also of driver's steering input.

\section{Simulation Models and Results}

\subsection{Single Track Vehicle Model}

To confirm the validity of above mentioned model matching control, simulation test based on the single track vehicle model was performed by using Modelica. First of all, we should handle time-varying linear state space system expressed by Equations (10) and (13). It was easy to describe time-varying state space system as Equations (10) and (13) by Modelica as mentioned below.

A new class of time-varying linear state space system was defined by using Modelica. For this purpose, the existing class of the linear state space system of Modelica Standard Library (MSL) was modified to release the constraint of variability of variables (i.e. by eliminating 'parameter' qualifier). The definition of the new class becomes as follow. 


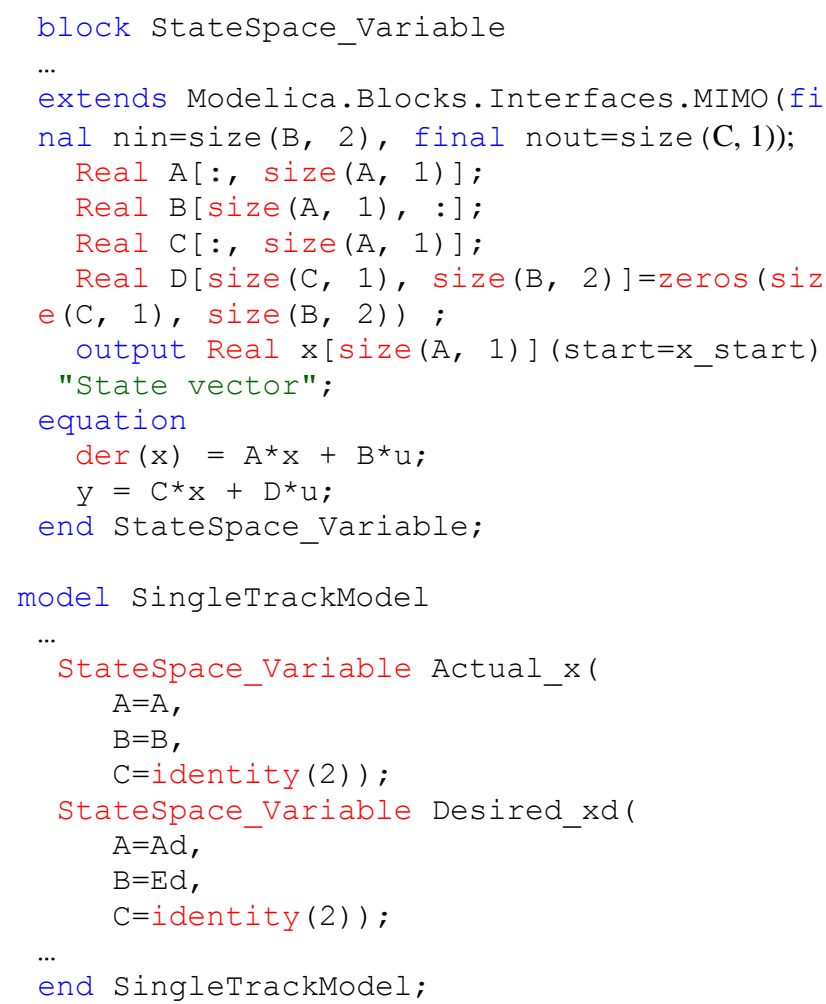

For comparison, the definition of the existing class of the linear state space system in MSL is as below.
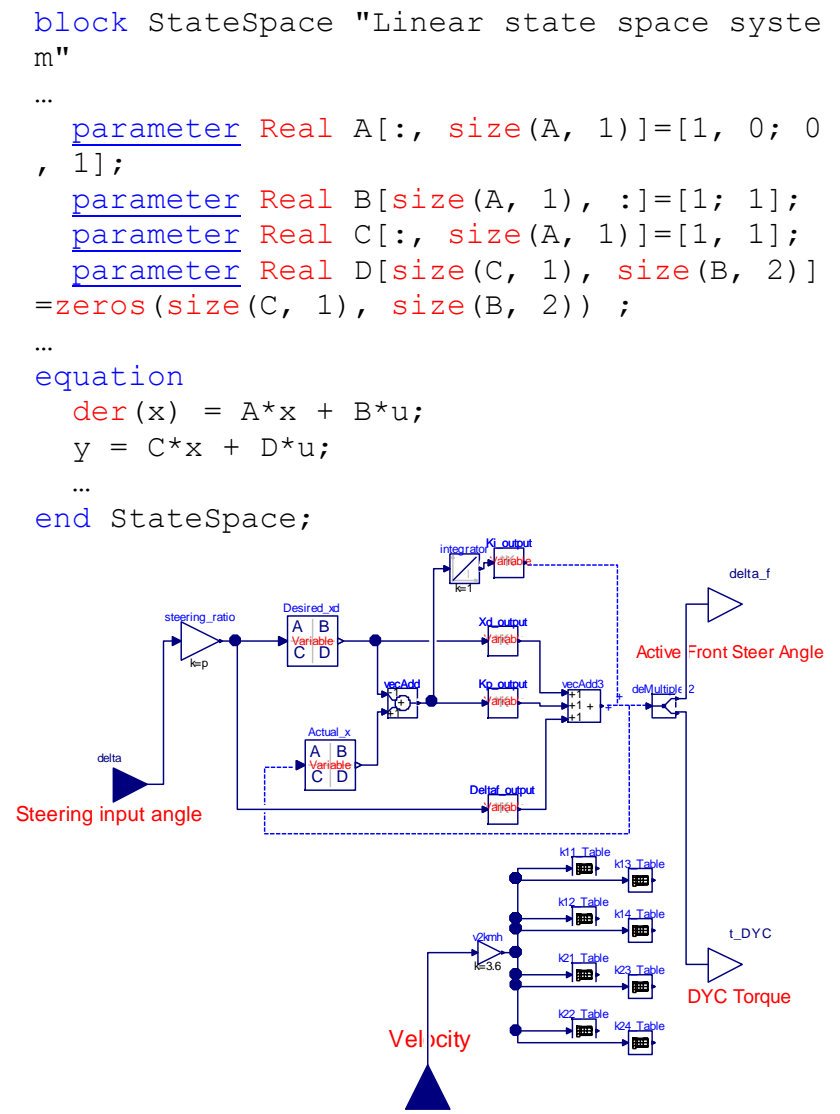

Figure 3. Modelica model of the controller

Figure 3 shows a diagram of the Modelica model of the model matching controller as defined by Equation
(28). The time varying linear state systems of both plant model and desired dynamics model as mentioned above are used in this model. Also 1D table elements are used to define the matrix gains which are dependent on the vehicle speed. Please note that it was impossible to write the model by connecting elements by normal Modelica 'connection' as shown by dashed lines in Figure 3. There occurred Modelica translator error by this way. If we connect the dashed lines as normal Modelica 'connection', then this model becomes under-constrained because the variable $S$ in the Equation (27) is not defined. To solve this problem, 'algorithm' section to calculate final value of $u$ was used in this model. (Dashed lines in Figure 3 indicate that there is additional summation of the signals just graphically. It is a little shortcoming of Modelica that all of the equations including 'algorithm' section cannot be seen directly in the graphical window.)

Figure 4 shows simulation results using the single track model. Vehicle accelerates from $10 \mathrm{~km} / \mathrm{h}$ to $100 \mathrm{~km} / \mathrm{h}$ between time $1 \mathrm{sec}$ to $10 \mathrm{sec}$. The steering input angle moves as $1 \mathrm{~Hz}$ sinusoidal curve. Desired dynamics was settled as $k_{\beta}=0.3$ and $k_{\gamma}=1$. $\tau_{\beta}$ and $\tau_{\gamma}$ are settled as corresponding value of cut-off frequency of $1.3 \mathrm{~Hz}$.

The results of vehicle slip angle and yaw rate are shown not only in the nominal plant but also there were some perturbation of vehicle mass $(\mathrm{M})$ and tire cornering power $(\mathrm{CP})$. In the nominal case, the results of slip angle and yaw rate were exactly matched with the desired values. It is shown that the model matching control has rather good robustness against the perturbation of the parameter M. But it is not so robust against the change of the plant parameter CP. The improvement of the robustness of the control should be a future research. 

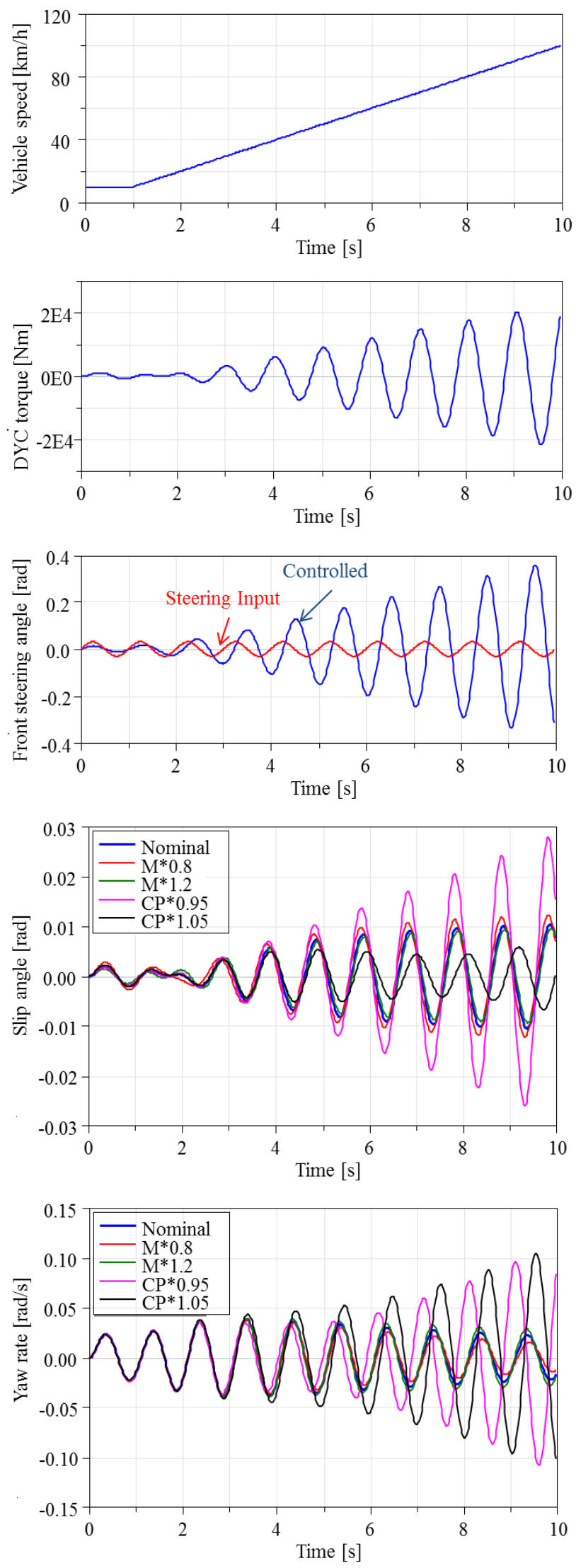

Figure 4. Plot of vehicle velocity and steering input angle

\subsection{Full vehicle model}

The full vehicle model as previous research (Hirano et al., 2013)(Hirano et al., 2014) was used for full-vehicle simulation. The model was developed based on Vehicle Dynamics Library of Modelica (Modelon, 2014) and was built as a full 3-dimentional (3D) multibody-dynamic system (MBS) model. Component models of control systems such as TVD gearbox, electric motor and inverter were added with the full vehicle model.
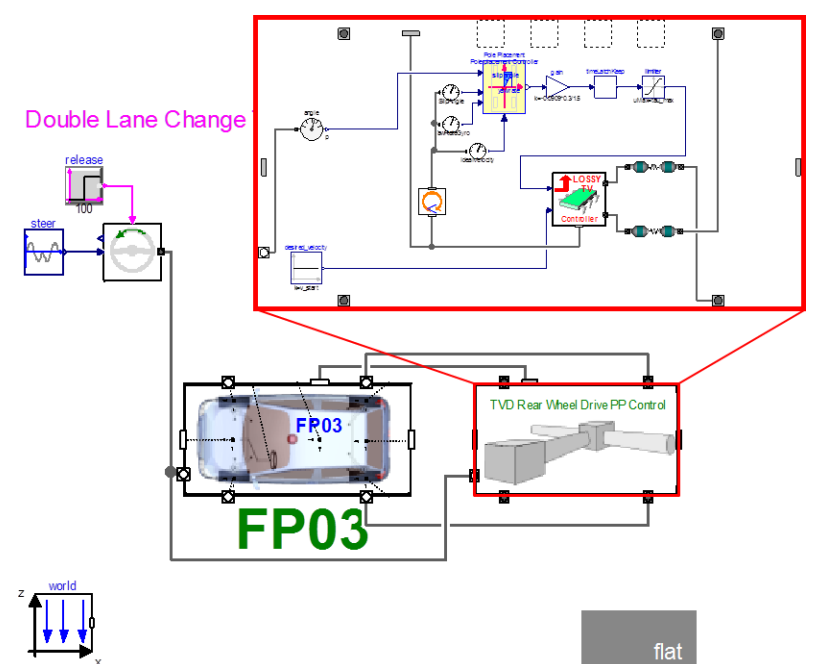

Figure 5. Structure of full vehicle test model

Figure 5 shows the top level of the model hierarchy of the full vehicle test model and also the power train model with the controller.

For the TVD gear train, a driveline structure referencing the MUTE project of the Technische Universität München (Höhn et al., 2013) was selected and the TVD model was constructed using Modelica Power Train Library (DLR, 2013). Figure 6 shows the configuration of the gear trains. Torque from the main motor is distributed equally to the left wheel and the right wheel through the differential gear. The torque distribution between the left wheel and the right wheel can be controlled by changing the torque input of the control motor.

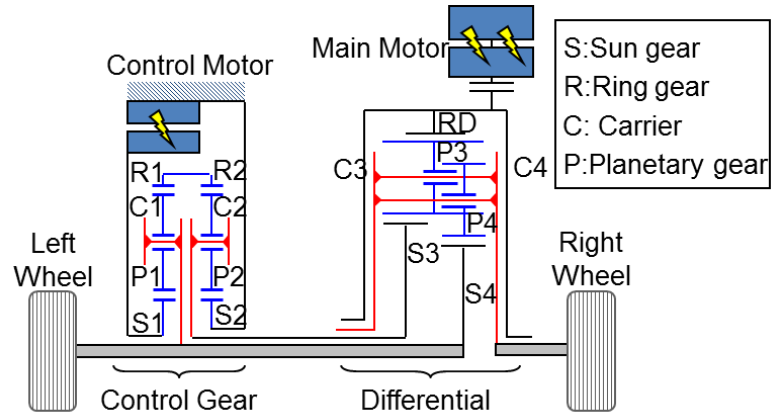

Figure 6. Torque vectoring differential (TVD) driveline 


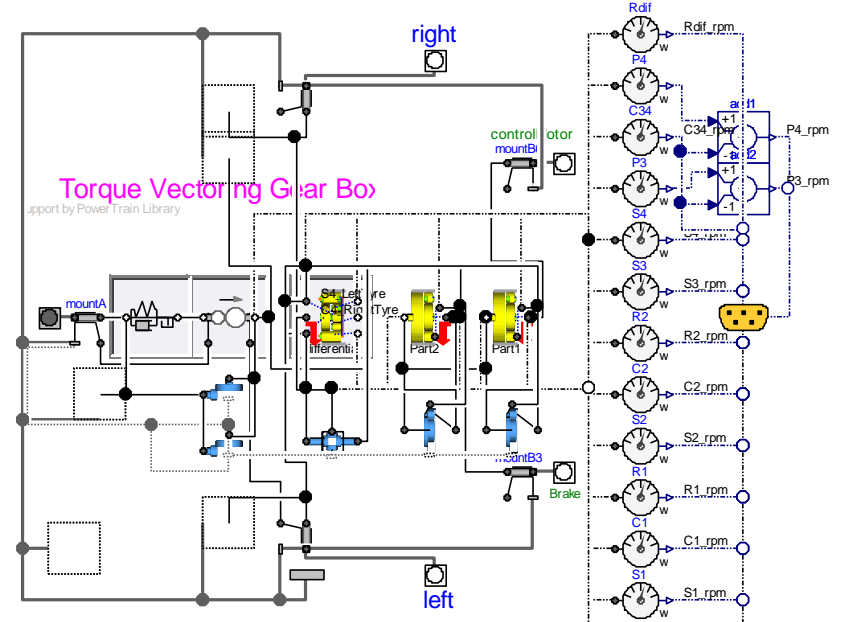

Figure 7. Modelica model of TVD gear train

Figure 7 shows a diagram of Modelica model of the torque vectoring gear train. The model is provided with elements that define the relational expression between the torque and speed of each gear engagement portion.

3D MBS model of suspension, steering and body were installed to calculate vehicle dynamics characteristics. Suspension model was constructed as an assembled model of each suspension linkage, joints and force elements such as spring, damper and bushing. Non-linear tire model based on 'Magic Formula' model (Pacejka02) was used to calculate combined lateral force and longitudinal force of each tire. Steering model considered the characteristics of viscous friction of steering gear box and steering shaft as well as steering shaft stiffness.

\subsection{Results of full vehicle simulation}
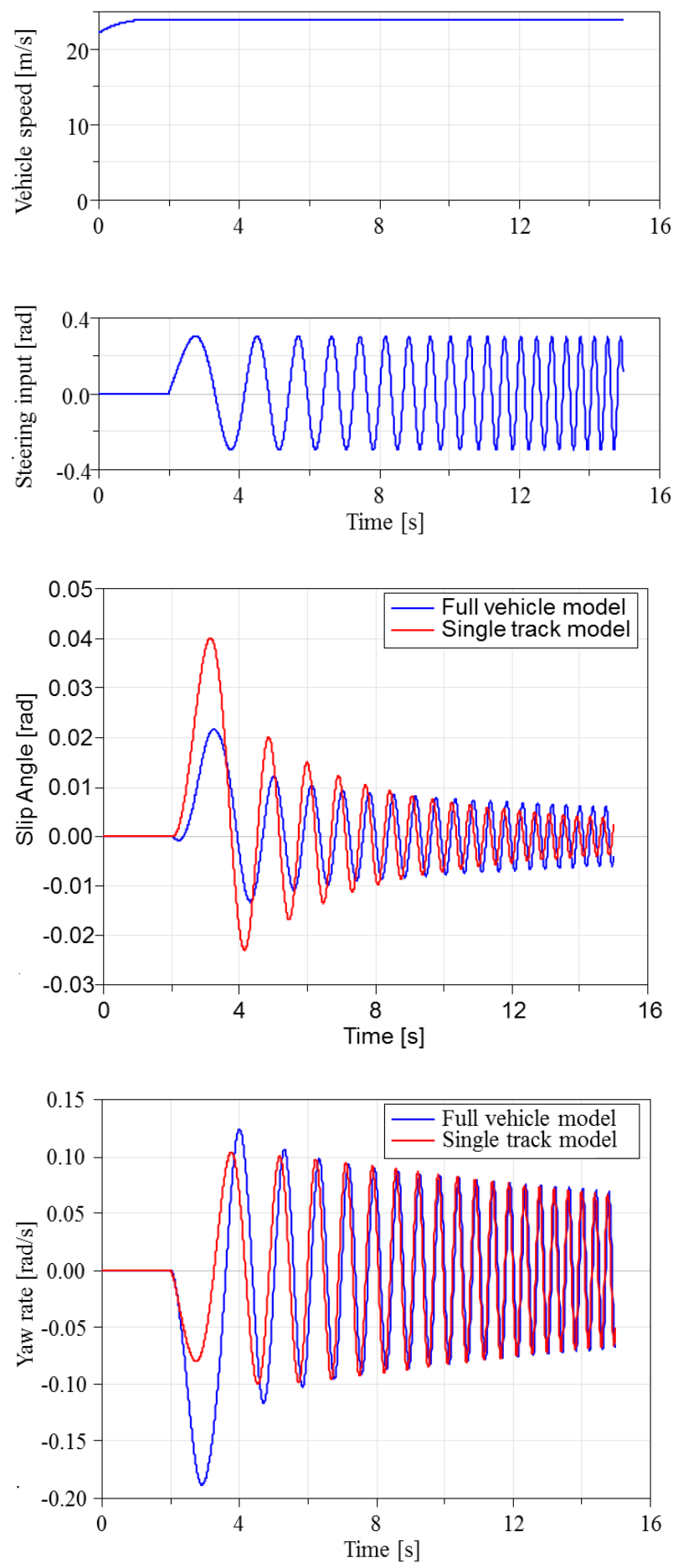

Figure 8. Comparison between full vehicle model and single track model

At first, the result of the full vehicle model and the single track model was compared in a case that no control was applied. Steering input angle was given as a sinusoidal sweep signal from $0.1 \mathrm{~Hz}$ to $5 \mathrm{~Hz}$ at constant vehicle speed $V=80[\mathrm{~km} / \mathrm{h}]$. Figure 8 shows the results of vehicle slip angle and yaw rate response. It is shown that some difference exists between the single 
track model and the full vehicle model especially in the low frequency response. The reason of this result is assumed that the approximation when used to derive the equation of the single track model was too big. Actually the Equation (2) and (3) about the Figure 1 should be

$$
\begin{gathered}
M \frac{d}{d t}\left(u \tan ^{-1} \beta+u \gamma\right) \cong 2 Y_{f} \cos \delta_{f}+2 Y_{r} \\
I_{z} \frac{d \gamma}{d t} \cong 2 l_{f} Y_{f} \cos \delta_{f}-2 l_{r} Y_{r}+N
\end{gathered}
$$

in precise. Also the non-linearity of the tire characteristics and effects of many losses and stiffness of mechanical parts are not considered in the single track model. This result indicates that we should be careful when designing controllers based on the single track model.

Next, a simulation emulating double lane change maneuver was performed. Though, in this case, a problem that the vehicle motion of the full vehicle model became unstable when applying the control law shown in Eq. (28). The reason was that by the default gain of the feed forward control parts, the controlled steering angle exceeded the actual physical limit and turned more than $6[\mathrm{rad}]$, that is, about $360[\mathrm{deg}]$. So the compensation for the feed forward parts was applied so that the controlled front steering angle will not be so different from the steering input angle. (Actually the feed forward parts were gained by 0.1.) After this modification, the vehicle response became stable in the actual case using the full vehicle model. Also for the feedback part, we should be careful to select the value of weight matrix element when designing LQR controller. Also the weight for the steering angle control was lowered than that for the DYC torque because of the physical limit of the steering angle. These problems may be solved by modifying the controller design from LQR to MPC (Model Predictive Control) which can consider the limitation of the actuators, but there would be a conflict of calculation time of the controller in such a case.

Figure 9 shows the results of the full vehicle simulation imitating the double lane change test by open-loop driver model. Though there seems necessity of further gain tuning, the modified model matching control seems to work to let the actual state variables trace the desired variables.

Also side wind test was simulated using the full vehicle model. Figure 10 shows the results. There is a side wind of $20[\mathrm{~m} / \mathrm{s}]$ while time $=2 \mathrm{sec}$ to $3.5 \mathrm{sec}$ when the vehicle is running at $120[\mathrm{~km} / \mathrm{h}]$ with fixed steering input angle of $0[\mathrm{rad}]$. The effect of the proposed control to stabilize both slip angle and yaw rate response against the side wind was shown.

For comparison, the result of the previous research in which design of the model matching control was done without considering the integral of the error (Hirano, 2016b) is shown in Figure 11. The new control (Figure 10) showed less steady state deviation and also better regulation of the state variables against the external disturbance as the side wind, though it is not perfect yet.
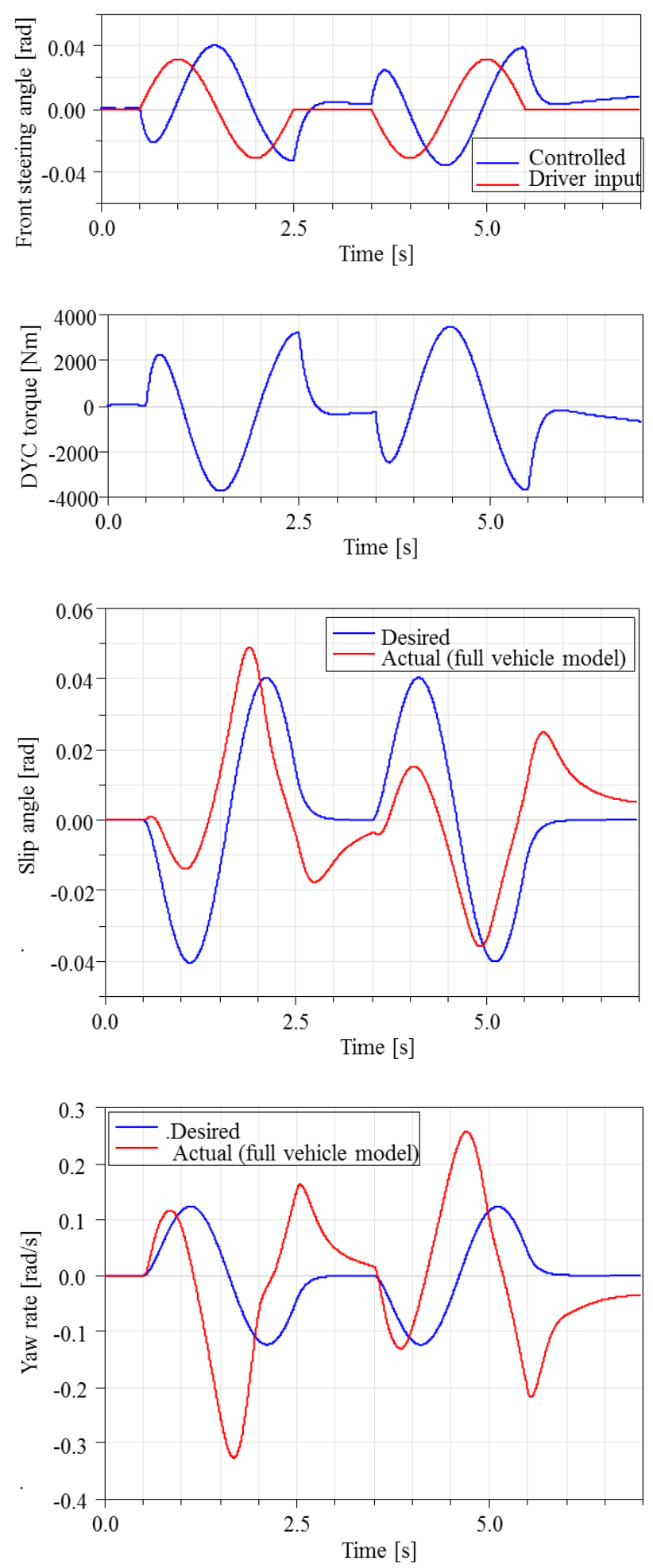

Figure 9. Full veheicle simulation result for double rane change test 

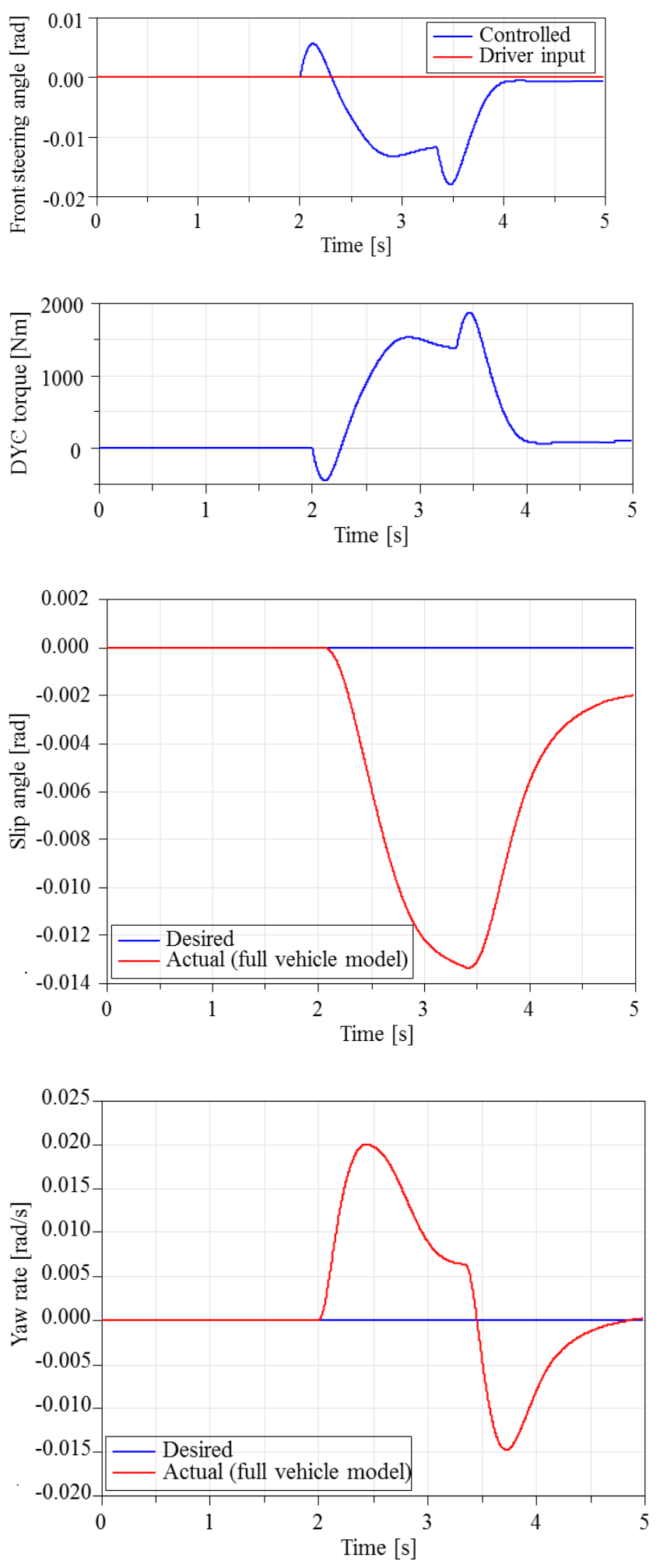

Figure 10. Full vehicle simulation result of side wind test
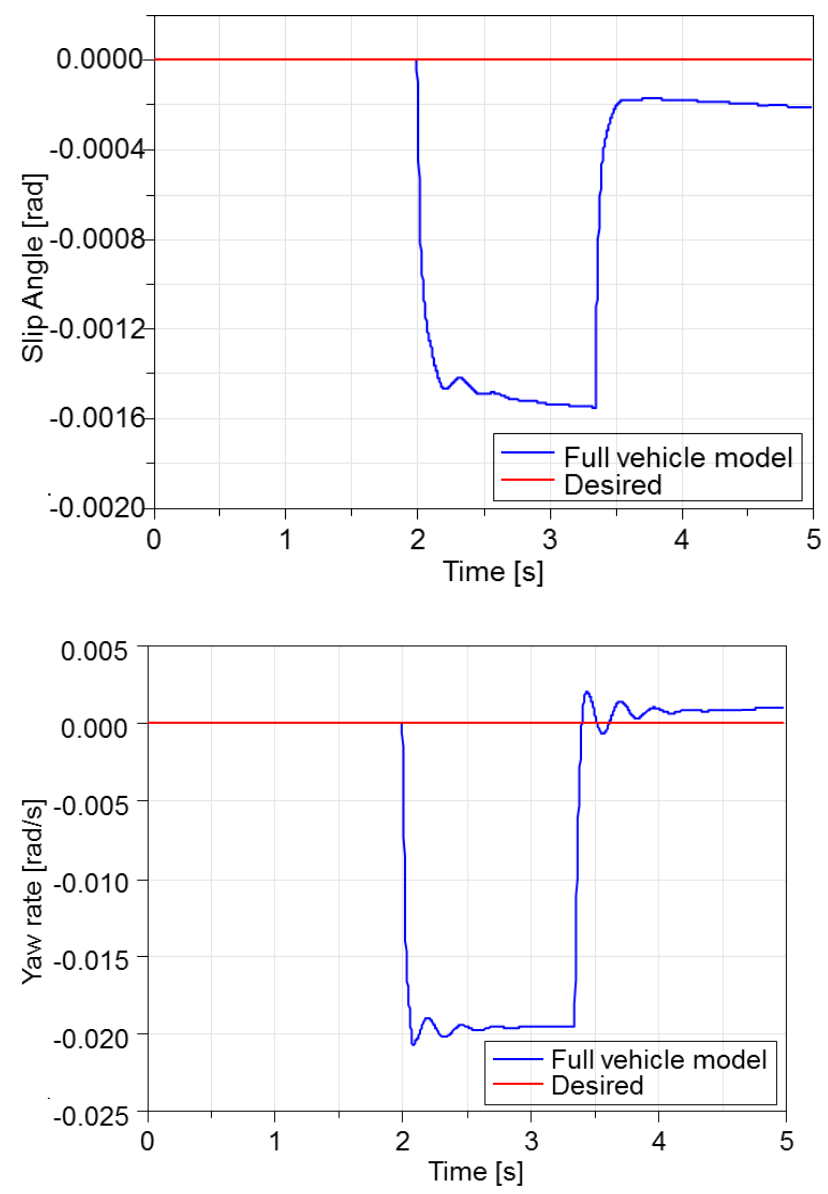

Figure 11. Side wind test result by previous control

\section{Conclusion}

A new integrated control of DYC and front steering angle was proposed using model matching control also considering the integral of the error. By simulations using both single track model and full vehicle model based on Modelica, the effect of the control was investigated. Also the limitation of control design based on the single track model was clarified by comparing the results of the simulation by both single track model and full vehicle model. Some know-how about controller design was also obtained from the full vehicle model simulation considering various limitations of the actual vehicle. Because of the limitation of the actual actuators and also neglected modeling errors, the results of the proposed control was not satisfactory.

On the other hand, Modelica was always powerful to express any kind of controllers as well as multiphysics full vehicle model. A new technique to expand Modelica model to write time-variant models was also introduced. 


\section{References}

M. Burgess, Torque vectoring. Lotus Engineering, 2009.

DLR(German Aerospace Center), Power Train Library Users Guide (Version 2.1.0), 2013

S. Efstathios, E. Velenis, and S. Longo, Model predictive torque vectoring control for electric vehicles near the limits of handling. European .Control Conference (ECC) 2015 IEEE, 2015.

Y. Hirano, S. Inoue and J. Ota, Model-based Development of Future Small EVs using Modelica, Proceedings of Modelica Conference 2014, 2014.

Y. Hirano, S. Inoue and J. Ota, Model Based Performance Development of a Future Small Electric Vehicle by Modelica, Proceedings of Modelica Conference2015, 2015.

Y. Hirano, Research of Model Matching Control of Torque Vectoring Differential Gear System, Proceedings of Japanese Modelica Conference2016, 2016a

Y. Hirano, Model Based Development of an Integrated Control of Front Steering and Torque Vectoring Differential Gear System, Proceedings of SICE2016, $2016 b$

B. Höhn et al., Torque Vectoring Driveline for Electric Vehicle, Proceedings of the FISITA 2012 World Automotive Congress, Vol. 191, pp. 585-593, 2013.

Modelon, A.B., Vehicle Dynamics library Users Guide (Version 1.8), 2014 